\title{
The Impact of Open and Distance Learning Material on Mindfulness Practices of Primary School Teachers
}

\section{Kumbukage Dona Ruwandika Lakmali Jayalath Perera*, Galappaththige Dayalatha Lekamge}

Department of Secondary and Tertiary Education, Faculty of Education, The Open University of Sri Lanka, Nawala, Nugegoda, Sri Lanka

Email: ^kdper@ou.ac.lk, gdlek@ou.ac.lk

How to cite this paper: Perera, K. D. R. L. J., \& Lekamge, G. D. (2021). The Impact of Open and Distance Learning Material on Mindfulness Practices of Primary School Teachers. Open Journal of Social Sciences, 9, 83-94.

https://doi.org/10.4236/jss.2021.95007

Received: April 3, 2021

Accepted: May 8, 2021

Published: May 11, 2021

Copyright $\odot 2021$ by author(s) and Scientific Research Publishing Inc. This work is licensed under the Creative Commons Attribution International License (CC BY 4.0).

http://creativecommons.org/licenses/by/4.0/

\section{(c) (i) Open Access}

\begin{abstract}
At present, in Sri Lanka, there are no programmes developed to train teachers in mindfulness through Open and Distance Learning (ODL) methods that can easily be distributed. The materials developed have been compiled into a short course titled "Mindfulness Practices for Primary Teachers" which will be offered to teachers. Before, offering the course to a larger audience, a pilot study was conducted. The objectives of this pilot study were; to produce learning materials (in module and AV format) using the OUSL resources, to deliver the course (Print, AV, on-line) by enrolling Sinhala and Tamil primary teachers in Western Province, to assess the impact and delivery of the material by using a variety of means during the course and after completing the course, and to make suggestions for further improvement of the material in line with the findings of the pilot study. Mixes of survey and action research designs were used. Two questionnaires for primary teachers and OUSL academics who are involved in conducting the course, a questionnaire for the two experts, their reflections, and a reflective journal for primary teachers were used as the primary data collection methods. Samples of 100 teachers, six OUSL academics, and the two experts/facilitators were selected to enroll and complete the pilot study of the programme. The data collected through the questionnaires was analysed quantitatively using percentages and content analysis was used to analyse qualitative data recorded in the reflective journals and collected through documents. It could be concluded that the short course on mindfulness practices had a positive impact on the primary teachers. They were able to change themselves, their students, and their teaching processes because of the short course. The training received by the OUSL academics from the experts leads them to re-think about their day-to-day practices. Fur-
\end{abstract}


ther, the implementation of the course should be streamlined to facilitate the teachers self-practices.

\section{Keywords}

Mindfulness Practices, Open and Distance Learning, Pilot Study, Primary

Teachers, Short Course

\section{Introduction}

Mindfulness is described as "the awareness that emerges through paying attention on purpose, in the present moment, and nonjudgmentally to the unfolding of experience moment by moment' (Kabat-Zinn, 2003: p. 144). Mindfulness has spread into the fields of psychology, health care, education, business, management, neuroscience, and governance.

Mindfulness, as a technique of mental training has become very popular due to its vast benefits in developing focus and attention, with improved abilities to cope with stress and anxiety. The enthusiasm towards this practice is growing exponentially. Large numbers of people are downloading mindfulness applications and taking part in short and long mindfulness training courses in several countries. Teacher training on mindfulness is becoming very popular in many countries as it has a profound impact on both teachers' and students' behaviour.

Western developed countries have surged forward in this aspect during the past 2 - 3 decades with the expanding scientific understanding on this subject. In addition to over 5000 documented research studies on mindfulness, many large-scale studies involving school children and university students are also underway. Notable amongst these is the Welcome Trust sponsored UK based longitudinal study which has recruited more than six thousand school students and nearly 2000 teachers in the UK and is expected to give reliable answers as to whether mindfulness has a positive effect on the mental health of teenagers. The lab-based studies will also provide critical insight into how mindfulness works. Given the great social and personal toll of mental illness, building up the evidence base for preventative interventions such as mindfulness is crucial.

The benefits of mindfulness practice, and its application in two key sectors in social development such as education and health, are well documented, globally. It is increasingly evident through research data that mindfulness improves health and well-being by reducing stress, anxiety and depression, and increasing motivation to make lifestyle changes. Further, by reviewing hundreds of studies, Greeson (2009) revealed that mindfulness training is associated with reduced emotional distress, more positive state of mind and improved quality of life.

There are many programmes developed targeting teachers and students and several models applied for design, development, and delivery of programmes. Training teachers in mindfulness using face-to-face methods alone has proved to 
be daunting, given the numbers and distribution of schools in all nine provinces in Sri Lanka. Similarly, effective mindfulness training of health care workers using conventional methods, across all provinces is equally challenging. However, there are no programmes for teachers related to mindfulness practices conducted through ODL methods. As this is a very important concept, programmes developed using ODL methods could be given a significant contribution to the Sri Lankan context.

At present, in Sri Lanka, there are no programmes developed to train teachers in mindfulness through Open and Distance Learning (ODL) methods, as web-based on-line material, or as Trainer of Trainers (ToT) teaching/learning packs that can easily be distributed. The latter becomes imperative, since, as mentioned, trainers in the form of teachers are scattered all over Sri Lanka and often in locations with poor electricity coverage and no internet access. Therefore, in addition to web-based material, creative printed material/content should be developed which teachers and health care workers can use with minimal resources. Once developed, methods to test the usefulness of such material and to assess impact of same become necessary.

If successful, this programme will pave the way for new models of mindfulness ToT study packs, which can be used in impoverished societies and challenged groups of people globally.

Phase 1 of this Project has already been completed with the assistance of Sri Lanka Association for the Advancement of Education and the Faculty of Education, The Open University of Sri Lanka (OUSL). The materials developed have been compiled into a short course titled "Mindfulness Practices for Primary Teachers" which will be offered to teachers in the school system. The duration of the course is 2 months and a mixed mode incorporating modules, Audio-Video (AV) and on-line material will be used as necessary for the delivery of the programme. Before, offering the course to a larger audience, it is a requirement to test the material by conducting a pilot study.

Therefore, the proposed research study (Phase II of the Project) aims at testing the specialised mindfulness lesson material developed in a modular format and AV material with primary school (Grade 1 - 5) teachers in the Western Province. "The mindfulness practices for primary teachers" curriculum was developed by two experienced mindfulness trainers/coaches and then translated into Sinhala and Tamil and developed into modular format with the assistance of the staff of the Faculty of Education, OUSL. Included within these mindfulness lessons are components on developing mindfulness practices among primary teachers, and applying them in the teaching-learning process with the children. There are print materials in a $\mathrm{CD}$, an audio and a number of face-to-face contact sessions provided as learner support in this course. A two-day trainer of trainer programme was conducted by the two experts with a number of practical sessions to familiarise with the content of the two modules and also to develop skills necessary to conduct the practical sessions with primary teachers. 


\section{Methodology}

Mixes of survey and action research designs were used as necessary in the present study. It is aware that the mindfulness practices of a person cannot be evaluated through a test. It is planned to conduct the course in the Colombo and Gampaha centres in Sinhala medium at the initial stages due to unavoidable circumstances and then pilot with the Tamil medium teachers. Specific objectives of the research study were:

- To produce learning materials (in module and AV format) using the OUSL resources.

- To deliver the course (Print, AV, on-line) by enrolling Sinhala and Tamil primary teachers in Western Province.

- To assess the impact and delivery of the material by using a variety of means during the course and after completing the course.

- To make suggestions for further improvement of the material in line with the findings of the pilot study.

Those components are considered as the survey part of this research.

In addition, the action research design had been used to develop the skills related to mindfulness practices among teachers. The experts applied different methods and activities during the contact sessions with primary teachers and come to a conclusion about them, which can be practiced at a later stage by the academic staff of the Faculty of Education when they play the role of the facilitators.

The impact of delivery including the teaching methods used and quality of the material developed and also the changes taken place in teachers during the study period were considered as major components of the study.

The sample of the pilot study consisted of 100 primary teachers in the Western Province selected in consultation with the Ministry of Education. It was expected that these 100 teachers would represent all categories of schools as well as advantaged and disadvantaged schools. The teachers were enrolled in the course in small groups (25 in each). To reduce variations, it was expected to employ a few trained personnel on mindfulness practices to conduct the course. During the pilot study period there were practical difficulties for experts to travel long distances. When this short course is offered to other provinces, those who had participated in the pilot study group will be used as trainers for the rest of the teachers.

The primary teachers were selected as the target group considering the huge impact they can place on the small children. When they are mindful in many aspects in their life, including their teaching-learning process, children will consider them as their models. Further, the close relationship the primary teachers can maintain with small children will pave the way for the changes expected to take place in children.

Experienced mindfulness experts will be employed for the delivery of the course. Each participant will be given two modules and a video and audio up- 
loaded to a CD. One face-to-face contact session of 6 hours duration per module will be conducted to provide hands-on-experience for the participants to familiarise with the mindfulness practices. The Colombo centre and Gampaha centre were used for implementing the pilot run of the course.

The duration of the pilot study was eight months, which include delivery of the course, assessment of the impact of the course, analysis of data and dissemination of findings of the study.

Two questionnaires for primary teachers in the short course and OUSL academics/course team who are involved in conducting the course, a questionnaire for experts who participated in the workshops and a reflective journal for primary teachers were used as the primary data collection methods in the present study. The questionnaire was administered at the beginning of the course and at the end of the course and the participants were asked to maintain a reflective journal during the two months period. Also, the reflections of the experts were collected at the beginning and at the end of the course.

The data collected through the questionnaires was analysed quantitatively using percentages and content analysis will be used to analyse qualitative data recorded in the reflective journals and collected through documents.

\section{Results and Discussion}

There were four objectives set for the second phase of the study. The first objective had to be achieved first to progress with the rest of the objectives. In the first phase, two experts completed two modules, learning journal, and other activities in consultation with the team members of the project. It was very encouraging to note that these experts conducted presentations to the project team at crucial stages of the module development and modified the sessions according to others views. Once three sessions of each module had been completed the translations into Sinhala and Tamil were started to save the time and expedite the work. Translators identified within the project team completed their work under the supervision of two experts who then edited the translations and took necessary action to maintain the consistency among three versions.

Objective One: Produce learning materials (in module and AV format) using the OUSL resources

Having completed the sessions, two training of trainers' programmes were conducted by the two experts for the OUSL academics. The second workshop was a more practical-oriented one and a production of a video and audio were planned in addition to training for academics as another outcome of this workshop. This workshop was conducted at the Centre for Educational Technology and Media (CETMe) Studio with the participation of two experts, project team and the OUSL academics who were interested in becoming trainers of the course. The Technical team and equipment of the CETMe were ready for the task. The technical team videoed the whole session, which lasted for more than three hours and asked the research team to arrange a time for editing the video with the CETMe Director. When the project team approached the Director for 
editing, they were informed that there is a technical error and they cannot run the video for editing. They tried to solve the problem by sending it to another place but failed. So, the finalisation of the video could not be completed due to a technical error taken place at the CETMe, which had jeopardised the whole activity.

Both team members and experts had spent nearly three hours to prepare this video and the project had no additional money to do it once again. Therefore, intention of producing a course with multi-media material had to be restricted only to the modules and the audio. The CDs were prepared with the support of CETMe incorporating written material and the audio. This whole process was collaborative and completed with team spirit though it was very discouraging that the first objective could be achieved only $75 \%$ percent according to the opinion of the project team. However, all the members believed the whole process provided a good learning experience for them and they valued the opportunity extended to participate in practical workshops.

The OUSL academics agreed (Table 1) that the methods used by the experts in the workshops were successful and the activities conducted were effective. They felt that the knowledge and skills of the experts were sufficient. However, the majority did not agree with the statement about the sufficiency of the physical resources to conduct the workshops. The majority agreed about the active

Table 1. Responses of OUSL academics about the training workshops.

\begin{tabular}{|c|c|c|c|c|c|}
\hline Statement & $\begin{array}{l}\text { Strongly } \\
\text { agree }\end{array}$ & Agree & $\begin{array}{l}\text { Neither } \\
\text { agree/nor } \\
\text { disagree }\end{array}$ & Disagree & $\begin{array}{l}\text { Strongly } \\
\text { disagree }\end{array}$ \\
\hline $\begin{array}{l}\text { 1. The methods used to conduct the training } \\
\text { sessions by the experts were successful. }\end{array}$ & & 4 & & & \\
\hline $\begin{array}{l}\text { 2. The activities employed by the experts } \\
\text { were very effective. }\end{array}$ & & 4 & & & \\
\hline $\begin{array}{l}\text { 3. The knowledge and skills of the experts } \\
\text { were sufficient. }\end{array}$ & 2 & 2 & & & \\
\hline $\begin{array}{l}\text { 4. The physical resources used for conduct } \\
\text { training sessions were sufficient. }\end{array}$ & & 1 & & 3 & \\
\hline $\begin{array}{l}\text { 5. Active participation in the training } \\
\text { sessions was visible. }\end{array}$ & & 3 & & 1 & \\
\hline $\begin{array}{l}\text { 6. Participants were highly motivated } \\
\text { during the training sessions }\end{array}$ & & 4 & & & \\
\hline $\begin{array}{l}\text { 7. There was a close interaction between } \\
\text { experts and participants. }\end{array}$ & & 3 & & 1 & \\
\hline $\begin{array}{l}\text { 8. The training sessions were very useful to } \\
\text { get a clear understanding about } \\
\text { mindfulness practices }\end{array}$ & & 2 & 1 & 1 & \\
\hline $\begin{array}{l}\text { 9. The training sessions were very } \\
\text { comprehensive. }\end{array}$ & & 2 & 1 & 1 & \\
\hline $\begin{array}{l}\text { 10. Training sessions improved our } \\
\text { confidence to work as trainers }\end{array}$ & & 2 & & 2 & \\
\hline
\end{tabular}


participation of academics and close interaction between experts and academics maintained in the workshops though they had diverse views on the usefulness and comprehensiveness of the training workshops. The majority disagreed about the impact on improving their confidence to work as trainers. The reasons for the negative responses could be identified through their open-ended responses. All of them thought that more sessions are needed if they wish to take the responsibility as trainers of this course. Due to practical nature of the course, they suggested that more resources must be provided at the OUSL centres for experts to conduct sessions. As such, it could be concluded that the production of the video was unsuccessful though training workshops had a positive impact on OUSL academics and the workshops should be continued.

Objective Two: Deliver the course (Print, AV, on-line) by enrolling Sinhala and Tamil primary teachers in Western Province

While engaging in the finalisation of modules, the participants for the course were selected with the support of Directors of Education, Western Province and Director of Primary Education of the Ministry of Education. The experts also owe a special commendation from the project for their support extended. An application was prepared and distributed among primary teachers in the Western Province with the support of those personnel. Even though it was originally planned to enroll about 100 students in two centres representing both media, the number of primary teachers applied for the Colombo was limited due to communication. Nearly 15 at Colombo centre and 60 at the Gampaha centre were registered for the Sinhala Medium course and all the applicants were given the course free of charge. Two-sessions of 6 hour sessions were planned at each centre and the experts, primary teachers and Acting Directors of the two centres were informed about the arrangements for the sessions. However, the CDs could be distributed only on the first session. The first one hour of the first session was set a part for the inauguration of the course for which either the coordinator or a project team member had participated. At the inauguration both the OUSL procedures, structure of the course and the completion of the learning journal had been explained to the participants. In addition, a questionnaire developed by the project team had been distributed to the participants of the course to reveal their existing understanding about the Mindfulness practices. A separate questionnaire was developed and hand over to two experts to get their views on the conduct of the course.

Objective Three: Assess the impact and delivery of the material by using a variety of means during the course and after completing the course

In order to reveal the impact of the course on the participants, their expectations about the course, reflections on the material and teaching-learning process and reflections on influence of the course will be considered. As this is conducted as an action research study, reflections at the beginning of the course and at the end of the course also will be considered.

Expectations of the Primary Teachers about the Mindfulness Course

Almost all the participants in Colombo and Gampaha centre have mentioned 
that this course would be beneficial and the expectations revealed by them could be grouped as follows:

\section{Change Themselves and Students}

Some of their responses are; to become a mindful person and to make their students mindful, to maintain and improve good mental health, to get the knowledge about mindfulness and to practice them, to practice mindfulness and bring them to the classroom, to practice mindfulness in self and students, to use mindfulness in personal life and in the classroom, to guide students and to make them aware about mindfulness, to live with mindfulness and to make other teachers aware about it, and the main expectation was to improve their knowledge, understanding and practice on mindfulness in the classroom to create an impact on students.

\section{Solve Problems in the Day-to-Day Life}

Some of their responses are; to promote mindfulness in the family and school, to solve problems in day to day life, to bring success into my life and be a light to others, and to cultivate and maintain mindfulness within the self.

\section{Create a Change in the Teaching Process}

Some of their responses are; to identify students properly in the teaching-learning process and to do a better teaching, to bring the techniques of promoting mindfulness to the teaching process, to know the techniques to be followed to pave the path and to give a further knowledge to students, and to get understanding about mindfulness and practice it in the classroom.

\section{Overall Improvement}

Some of their responses are; to increase the quality of education through mindfulness and to have a better classroom.

\section{The Reflection of the Two Experts about the Conduct of the Course}

The two experts who had been involved in the conduct of the course were asked to reflect on the introduction session of the course, introduction to the mindfulness, teaching methods used by them, strategies used to get the active participation of teachers, behavioural changes observed by them, the role played by the two modules and overall impression of the teachers who followed the course.

Introduction session of the course: This part was done by two lecturers in the Department and both experts revealed that this provided a very good platform for teachers to be familiar with the distance and on-line practices of the OUSL. As they further revealed, the introduction to the two modules and the Audio was also impressive and resulted in improving the motivation of teachers.

Introduction to the Mindfulness: It was interesting to reveal that the two experts had used a variety of strategies to introduce the mindfulness to the primary teachers. They invited teachers to reflect upon their level of mindfulness in their day to daily lives, which may have provided a very simulative beginning for the sessions. As they revealed teachers had linked them to family as well as school matters and the majority had a moderate level of understanding on mindfulness.

Expert A: "The introduction was very clear, participatory and engaging". 
Expert B: "The teachers could grasp the definition and conceptual basis of mindfulness in an accessible and simple manner".

The above reflections illustrate the positive feelings of experts on the introduction.

Teaching methods used by the experts: One expert had listed the strategies to incorporate lectures, experiential sessions, sharing reflections and art exercises. The other focused more on the practical approaches such as guided meditation, experiential activities, small videos, Art and craft, self-reflection and gratitude training etc. some of which might be unique to the mindfulness practices.

Primary teachers willingly acknowledged the methods applied by the experts. The following viewpoints clearly explain the impression of primary teachers:

Expert A: "Group activities enabled teachers to interact and share experiences and tips. This created a sense of camaraderie and mutual support. Using methods like arts and crafts made the sessions fun and engaging for the teachers. They were able to directly witness and understand the impacts that the mindfulness activities introduced during the course would have on their children".

Expert B: "Teachers' feedback was positive. There was a fair amount of sharing. Many had experience about the methods we used".

The experts further revealed that the diversity of methods they had used kept the teachers engaged in the activities.

However, there were some negative outcomes observed by them.

"There was some misunderstanding when it came to the models, for example "auto pilot mode". It was evident that a verbal explanation may not have been sufficient based on questions raised by teachers". These reflections reveal the importance of using videos and audios supplementary to face-to-face.

The methods used by the two experts to maintain continuous participation of teachers were as follows:

Expert A: "The teachers had to actively engage and participate from the start. Some activities required teamwork and sharing such as back-to-back breathing and reflections on gratitude etc.".

Expert B: "We would call for volunteers and then if there was a reluctant to step forward we would ask particular persons to let us have their feedback. They were also given the opportunity to lead activities if they volunteered and wanted to".

It seems that active engagement, participation, leadership are some of the effective strategies used by the experts during face-to-face contact sessions.

The reactions of the primary teachers to the strategies used by the experts were positive:

Expert B: "Teachers commented on how the activities used during the course could be easily used in their classrooms with their primary students".

Expert A: "As it was a semi-formal format, teachers were willing to openly share their thoughts, insights and experiences. They were able to test activities in their home environments between the first and second workshops".

Evaluation of the face-to-face sessions: It seemed that the experts had used a 
variety of methods for evaluation of primary teachers' achievements. They included self-reflections and also peer sharing/reflection at the face-to-face sessions, completion of an OUSL questionnaire at the beginning and end of the course and a mindfulness journal for the duration of the trial in order to record cognitive and behavioural changes. However, during the sessions, experts felt that the time allocated to assess behaviour changes is limited though many reported incorporating the practice. Development of mindfulness practices takes time and cannot be assessed like the other cognitive outcomes. It requires a sufficient time to internalise mindfulness practices and make it a part of day-to-day practices.

The benefits achieved through modules: Both experts mentioned that the face-to-face sessions provided a very good introduction to the modules and the teachers needed time to go through the modules. The two modules and the exercises included in the modules would further support the teachers to establish what they have learned during face-to-face sessions.

Expert A: "The teachers pointed out how mindfulness can be an effective classroom management tool and how activities like the mind jar, gratitude visualisation, "anchor as breath" and back-to-back breathing can be used to create a conducive learning environment and improve student behaviour".

Expert B: "Effective and enjoyable. There is a need to contact teachers either on-line or face-to-face. Twice a week is preferable particularly for answering questions that might come up".

The reflections of the two experts provided a very good evidence for the effectiveness of the modules as well as the success of the teaching and evaluation methods used by the experts with the primary school teachers. Further, out of the two centres one centre (Colombo) had a lesser number of participants than the other centre, which was found as a facilitating factor for mindfulness practices by the two experts.

\section{Teaching Sessions}

It could be revealed that the reflections of primary teachers on the teaching sessions of the course were in the same lines as their facilitators. Some of their responses are; the support given through the teaching sessions is very important, the teaching sessions are motivating and encouraging, the guidance provided by the teachers to solve issues is very useful, the activities conducted during the sessions are very relevant, and the activities are motivating and facilitating.

\section{Modules and Audio-Video Materials}

While following the course the teachers reflected on the modules and AV material they had been using as follows. the ability to read the learning materials is very important, the usefulness of the video materials to increase the understanding about the concepts is very important, the usefulness of the audio materials to increase the understanding about the concepts is very important, the relevance of the reflective journal to increase the understanding of the concepts is very important, learning materials are pleasant, audio materials are pleasant, and video materials are pleasant. These reflections reveal that the participants had a 
positive impression about the two modules and the AV material.

\section{Reflections of Primary Teachers about the Impact of the Course}

It could be noted that several participants had not submitted the reflections at the end of the course. However, the analysis of the reflective journals submitted revealed that the course had a great impact on them. These reflections further revealed that most of them were able to realise their expectations, which may have resulted in creating a positive change in their students also. Some of their responses are; "I felt very free and pleasure as it has become a daily habit", "I was able to get rid of angry", "I learnt to think the things in life moderately", "I change my mindset positively when working with kids", and "Mind jar and mindful walking activities are interesting which lead to create a change in my teaching".

Through the analysis of the reflections of the two experts and primary teachers, it could be concluded that the course had met its expectations.

Problems Encountered by the Course Coordinators, Experts, and Primary Teachers

The course could be advertised only through the web and through the officials of the Ministry of Education. Therefore, course coordinators had difficulties in getting down the applications for the course through the Directors of the Ministry of education. Even though the Ministry officials expressed their willingness to join the course, their participation was limited. They could meet the participants only two times during the two-sessions and the reflective journals had to be collected after one month from the second session. This created problems in collecting the reflective journals from the primary teachers at the end of the course, which created limitations in the data collection.

According to the primary teachers, they did not have sufficient time to practice mindfulness practices in the classroom as well as in their day-to-day life. The two-sessions of the course were conducted at two-week intervals and the teachers felt that they needed more contact with the facilitators to internalise the changes into their behaviour. As they revealed the mindfulness practices can be strengthened through interactions and self-practices for which they had limited opportunities through the programme. Further, the teachers had a limited number of AV material, which were found from You-tube and other web sites and were in English, which may have led to limit their understanding on mindfulness practices.

Objective Four: Make suggestions for further improvement of the material in line with the findings of the pilot study

There were several suggestions made by them to the conduct of the course in an effective manner.

Expert B: Duration of the course (two months) should be expanded and a mechanism to review progress should be introduced.

Expert A: Two face-to-face sessions are not sufficient to cultivate mindfulness practices. Using videos and clips to support the explanation of models might be beneficial. Teachers also need time to practice doing mindfulness sessions and 
receive feedback from the facilitators and peers on technique and methodology.

Their main suggestions were related to increase the number of contact sessions, continuation of contact via on-line and extension of the time duration of the course.

The primary teachers suggested that the programmes should be introduced to all the schools in Sri Lanka. According to them, the understanding of mindfulness should be increased more. Further, they suggested having a specific time slot for this programme. In addition, they suggested having more group activities and more training sessions.

\section{Conclusion}

At the end of the study, it could be concluded that the short course on mindfulness practices had a positive impact on the primary teachers. They were able to change themselves, their students, and their teaching processes because of the short course. However, the course was mainly implemented using modules and face-to-face sessions, which was a deviation from the primary intention of the course. The primary objective was to introduce the course on-line and supplement it with some face-to-face sessions. However, due to limited funds available (re-production was not possible) and the technical failure experienced by the course team in producing AV material, the implementation methodologies had to be changed. The training received by the course team from the experts leads them to re-think about their day-to-day practices. Further, the implementation of the course should be streamlined to facilitate the self-practices of teachers. The course team of the opinion that the Tamil medium course also has to be pilot tested before offering the course for all media as a short course.

\section{Acknowledgements}

The research team would like to highly acknowledge the guidance and support received from Dr. Tara De Mel and the assistance received from the officials of the Ministry of Education, Sri Lanka.

\section{Conflicts of Interest}

The authors declare no conflicts of interest regarding the publication of this paper.

\section{References}

Greeson, J. M. (2009). Mindfulness Research Update: 2008. Complementary Health Practice Review, 14, 10-18. https://doi.org/10.1177/1533210108329862

Kabat-Zinn, J. (2003). Mindfulness-Based Interventions in Context: Past, Present, and Future. Clinical Psychology-Science and Practice, 10, 144-156.

https://doi.org/10.1093/clipsy.bpg016 